\title{
NUTRIENTES NO SOLO E NA SOLUÇÃO DO SOLO NA CITRICULTURA
}

FER TIRRIGADA POR GOTEJAMENTO

Doi:http://dx.doi.org/10.1590/1809-4430-Eng.Agric.v35n3p 484-493/2015

\section{THAIS R. DE SOUZA ${ }^{1}$, DIÓGENES M. BARDIVIESSO ${ }^{2}$, THOMAS F. DE ANDRADE ${ }^{3}$, ROBERTO L. VILLAS BÔAS ${ }^{4}$}

\begin{abstract}
RESUMO: A análise da solução do solo vem mostrando-se um método promissor de diagnóstico da fertilidade de solo, principalmente em sistemas de fertirrigação. O objetivo deste estudo foi avaliar o comportamento dos nutrientes no solo e na solução do solo, quando fornecidas cinco doses de $\mathrm{N}, \mathrm{P}_{2} \mathrm{O}_{5}$ e $\mathrm{K}_{2} \mathrm{O}(0 ; 25 ; 50 ; 100$ e $200 \%$ da dose recomendada) via fertirrigação. O experimento foi conduzido por três safras em laranjeiras Valência enxertadas sobre citrumelo Swingle. O solo e a solução do solo foram coletados ao final do período de fertirrigação de cada safra. As doses de N, $\mathrm{P}_{2} \mathrm{O}_{5}$ e $\mathrm{K}_{2} \mathrm{O}$ aplicadas proporcionaram redução nos valores de $\mathrm{pH}$ do solo e da solução do solo, porém a variação dos valores de $\mathrm{pH}$ da solução do solo foi 3,5 vezes maior que a observada no solo. Para o P, esta variação ficou em torno de 65 vezes e, para o K, foi maior que 100 vezes. Os teores de $\mathrm{P}$ e $\mathrm{K}$ apresentaram comportamento semelhante no solo e na solução do solo, com valores de correlação de 0,87 e 0,97, respectivamente, e ambos os teores de $\mathrm{P}$ e K aumentaram em função das doses de fertilizantes. Os resultados sugerem que a análise da solução do solo pode ser utilizada como ferramenta para estimar os valores de $\mathrm{pH}, \mathrm{P}, \mathrm{K}, \mathrm{Mn}$ e $\mathrm{Zn}$ no solo, e também para determinar a disponibilidade de nitrogênio em sistemas de fertirrigação na citricultura.
\end{abstract}

PALAVRAS-CHAVE: Citrus sinensis, extrator de solução do solo, fertilidade do solo.

\section{SOIL AND SOIL SOLUTION NUTRIENT CONTENT IN DRIP FERTIGATED CITRUS}

ABSTRACT: Soil solution analysis has shown to be a promising method for soil fertility diagnosis, mainly in fertigation systems. The objective of this study was to evaluate the behavior of nutrients in soil and soil solution by supplying five doses of $\mathrm{N}, \mathrm{P}_{2} \mathrm{O}_{5}$ and $\mathrm{K}_{2} \mathrm{O}(0,25,50,100$ and $200 \%$ of the recommended dose) via fertigation. The experiment was conducted during three seasons in "Valencia" sweet orange on Swingle rootstock. Soil and soil solution samples were collected after fertigation at each season. The supplied doses of $\mathrm{N}, \mathrm{P}_{2} \mathrm{O}_{5}$ and $\mathrm{K}_{2} \mathrm{O}$ decreased $\mathrm{pH}$ in both soil and soil solution; however soil solution variation was 3.5 times higher. Such differences were also observed for $\mathrm{P}$ and $\mathrm{K}$ contents, which were 65 times and above 100 times higher than in soil, respectively. These nutrients had similar behavior in both environments with correlation values of 0.87 and 0.97 respectively, and both values of $\mathrm{P}$ and $\mathrm{K}$ increased according to fertilizer dose. The results suggested that soil solution analysis can be used as a tool to estimate values of $\mathrm{pH}, \mathrm{P}, \mathrm{K}, \mathrm{Mn}$ and $\mathrm{Zn}$ in the soil, as well as to determine nitrogen availability in citrus production under fertigation systems.

KEYWORDS: Citrus sinensis, Soil solution extractor, Soil fertility.

\footnotetext{
${ }^{1}$ Engenheira Agrônoma, Pesquisadora Doutora, Centro de Pesquisa Hanninghof Yara, Dülmen/Alemanha, Fone: (+49) 2594798192, thais.desouza@yara.com

${ }^{2}$ Engenheiro A grônomo, Prof. Mestre, Departamento de Agronomia, UEMS/Cassilândia - MS, bardiviesso@uems.br

${ }^{3}$ Engenheiro A grônomo, Doutorando em Irrigação e Drenagem, Departamento de Solos e Recursos Ambientais, UNESP/

Botucatu - SP, tfandrade@fca.unesp.br

${ }^{4}$ Engenheiro A grônomo, Prof. Doutor, Departamento de Solos e Recursos Ambientais, UNESP/Botucatu - SP, rlvboas@fca.unesp.br 


\section{INTRODUÇÃO}

A irrigação tem sido adotada de forma crescente pelos citricultores nos últimos anos, devido, principalmente, à ele vação da produtividade, à melhoria da qualidade dos frutos e à possibilidade de produção fora de época. COELHO et al. (2011) comentam que a irrigação pode proporcionar aumentos de 35 a $75 \%$ na produção de frutos cítricos. Grande parte dos citricultores que adotam a irrigação vem empregando o sistema de irrigação localizada por gotejamento, associado à fertirrigação. Uma das principais vantagens da fertirrigação é a melhoria da eficiência da adubação, pois possibilita maior parcelamento dos nutrientes, reduzindo as perdas, além de permitir melhor ajuste da adubação de acordo com a necessidade da cultura (OLIVEIRA et al., 2011).

Atualmente, a recomendação de adubação na citricultura brasileira é baseada na análise de solo e foliar (QUAGGIO et al., 2005). No entanto, tais métodos demandam certa quantidade de tempo, desde a coleta das amostras até à obtenção dos resultados, o que, em muitos casos, limita a adequação da adubação, principalmente em sistemas de fertirrigação, que permitem ajustes frequentes. Este fato tem despertado o interesse pelo uso de ferramentas diagnósticas auxiliares, que possibilitem a obtenção de informações mais rápidas sobre as condições químicas do solo e o estado nutricional das plantas.

A utilização de extratores de cápsula porosa para extrair a solução do solo vem sendo bastante difundida, principalmente por representar as condições reais de umidade do solo e por avaliar os elementos disponíveis às plantas. Além disso, possibilita a coleta da solução diretamente no campo, de forma não destrutiva, permitindo repetição no mesmo local, o que favorece o monitoramento por longos períodos (SOUZA et al., 2013). Muitos estudos vêm sendo conduzidos no sentido de identificar e corrigir problemas causados por desequilíbrios nutricionais, processos de salinização e perdas por lixiviação, utilizando como referência a análise da solução do solo (MEDEIROS et al., 2012; OLIVEIRA et al., 2011; SILVA JR. et al., 2010). De acordo com SOUZA et al. (2012), a análise da solução do solo, obtida por extratores de cápsula porosa, pode ser considerada uma ferramenta auxiliar para monitorar e avaliar a disponibilidade de nutrientes em sistemas de fertirrigação na citricultura.

Pesquisas relacionadas com a concentração e o monitoramento dos nutrientes na solução do solo são escassas e necessitam de especial atenção por se tratar de um método promissor de diagnóstico da fertilidade de solo, principalmente em sistemas de fertirrigação. Como os nutrientes em solução são os efetivamente disponíveis para as plantas e estão em equilíbrio com a fase sólida do solo, o objetivo deste estudo foi avaliar o comportamento dos nutrientes no solo e na solução do solo, quando fornecidas cinco doses de $\mathrm{N}, \mathrm{P}_{2} \mathrm{O}_{5}$ e $\mathrm{K}_{2} \mathrm{O}$ via fertirrigação, na citricultura.

\section{MATERIAL E MÉTODOS}

O experimento foi conduzido no município de Reginópolis-SP, à latitude de $21^{\circ} 49^{\prime} 45^{\prime \prime}$ sul, longitude de $49^{\circ} 08^{\prime} 27^{\prime \prime}$ oeste e altitude de $460 \mathrm{~m}$. De acordo com a classificação de Köppen, o clima da região é do tipo $\mathrm{Cwa}$, clima mesotérmico tropical de altitude, com inverno seco e temperatura média do mês mais quente superior a $22^{\circ} \mathrm{C}$. O pomar era composto por laranjeiras da variedade Valência [Citrus sinensis (L.) Osbeck], enxertadas sobre citrumelo Swingle [Citrus paradise Macfad. x Poncirus trifoliata (L.) Raf.], com população de 357 plantas por ha. O experimento teve início em 2007, quando as plantas tinham quatro anos de idade, e foi conduzido por quatro safras (2007-2008 a 2010-2011). Porém, os dados de solo e solução do solo apresentados neste trabalho foram referentes a três safras (2007-2008, 2008-2009 e 2010-2011). Na safra de 2009-2010, não foi feita coleta de solução do solo próxima da coleta de solo.

Antes do início do experimento, amostras de solo, retiradas na camada de 0-20 cm da área experimental, tinham as seguintes características químicas, determinadas segundo os métodos descritos em RAIJ et al. (2001): 5,0 de $\mathrm{pH}\left(\mathrm{CaCl}_{2}\right) ; 9 \mathrm{~g} \mathrm{dm}^{-3}$ de $\mathrm{MO} ; 57 \mathrm{mg} \mathrm{dm}^{-3}$ de P (resina); 21; 3; 14; 5; 22 e 44 mmol $_{\mathrm{c}} \mathrm{dm}^{-3}$ de $\mathrm{H}+\mathrm{Al}, \mathrm{K}, \mathrm{Ca}, \mathrm{Mg}, \mathrm{SB}, \mathrm{CTC}$, respectivamente, e $51 \%$ de saturação por bases. O solo foi classificado como Argissolo Vermelho-Amarelo distrófico, sendo que, para a 
profundidade de $0-40 \mathrm{~cm}$, foi considerado arenoso, com teor de areia superior a $81 \%$ (SANTOS et al., 2006)

O delineamento experimental utilizado foi o de blocos ao acaso, com quatro repetições. Os tratamentos foram constituídos de cinco doses de $\mathrm{N}, \mathrm{P}_{2} \mathrm{O}_{5}$ e $\mathrm{K}_{2} \mathrm{O}(0 ; 25 ; 50 ; 100$ e $200 \%$ da dose recomendada) aplicadas via fertirrigação. Nas três primeiras safras (2007-2008 a 2009-2010), a dose $100 \%$ correspondeu a $140 ; 40$ e $130 \mathrm{~kg} \mathrm{ha}^{-1}$ por ano de $\mathrm{N}, \mathrm{P}_{2} \mathrm{O}_{5}$ e $\mathrm{K}_{2} \mathrm{O}$, respectivamente. $\mathrm{Na}$ safra de 2010-2011, a dose $100 \%$ correspondeu a 220 e $130 \mathrm{~kg} \mathrm{ha}^{-1}$ por ano de $\mathrm{N}$ e $\mathrm{K}_{2} \mathrm{O}$, respectivamente. As doses foram estabelecidas com base no histórico de análises de solo e folha, e também na produtividade esperada (QUAGGIO et al., 2005). As fertirrigações foram efetuadas desde o início de setembro até o final do mês de abril de cada safra, em duas aplicações semanais. As fontes de $\mathrm{N}, \mathrm{P}_{2} \mathrm{O}_{5}$ e $\mathrm{K}_{2} \mathrm{O}$ utilizadas foram nitrato de amônio, ácido fosfórico e cloreto de potássio, respectivamente.

A calagem, em cada safra, foi realizada no mês de setembro com o intuito de elevar a saturação por bases a $70 \%$. O cálculo da necessidade de calagem teve por base o resultado da análise de solo, coletado ao final do período de fertirrigação, para o tratamento $100 \%$ da aplicação de $\mathrm{N}, \mathrm{P}_{2} \mathrm{O}_{5}$ e $\mathrm{K}_{2} \mathrm{O}$.

O sistema de irrigação utilizado foi por gotejamento. As parcelas possuíam uma linha de tubo gotejador, instalada ao lado do tronco das árvores, paralelamente à linha de plantio. A linha de gotejo era composta por emissores autocompensantes, com vazão de $2,3 \mathrm{~L} \mathrm{~h}^{-1}$, espaçados entre si a $0,6 \mathrm{~m}$, submetidos à pressão de serviço entre 5 e $30 \mathrm{~m}$ c.a., e proporcionavam faixa de área molhada de aproximadamente $0,8 \mathrm{~m}$ de largura. A injeção dos fertilizantes no sistema de irrigação foi feita com o injetor tipo "Venturi".

O manejo da irrigação foi realizado com base na medida diária da evaporação, por meio do tanque Classe A, obtendo-se a evapotranspiração potencial (ETP) e a evapotranspiração da cultura (ETc), conforme ALLEN et al. (1998). A umidade do solo também foi monitorada por três baterias de tensiômetros, sendo cada bateria formada por três tensiômetros instalados nas profundidades de 30,60 e $90 \mathrm{~cm}$. As precipitações ocorridas nas safras de 2007-2008, 2008-2009, 2009-2010 e 20102011 foram de $1.215 ; 1.019 ; 1.197$ e $1.560 \mathrm{~mm}$, respectivamente.

As parcelas experimentais foram constituídas por três linhas com doze plantas, sendo que as dez plantas da linha central foram designadas úteis para as avaliações das variáveis de interesse do experimento. As duas linhas laterais e as duas plantas nos extremos da linha central formavam a bordadura, totalizando 36 árvores por parcela. $O$ experimento ocupava uma área de aproximad amente 2 ha.

A amostragem do solo foi realizada após o término das fertirrigações, no mês de maio dos anos de 2008, 2009 e 2011, e nas camadas de 0-20 e 20-40 cm, sendo estas amostras retiradas na projeção da copa e a $20 \mathrm{~cm}$ do emissor. Foram coletadas 10 subamostras por parcela que, após a homogeneização, deram origem a uma amostra composta. Os valores dos macro e micronutrientes foram determinados de acordo com a metodologia descrita por RAIJ et al. (2001). Os resultados apresentados foram referentes aos valores médios observados nas camadas de 0-20 e 20-40 cm (camada 0-40 cm).

A solução do solo foi extraída com o auxílio de extratores de solução de cápsula porosa. Cada extrator era composto de um tubo de PVC (12,7 mm de diâmetro), conectado, na extremidade inferior, a uma cápsula de cerâmica porosa (60 mm de comprimento e $19 \mathrm{~mm}$ de diâmetro). Na extremidade superior, o extrator era vedado com rolha atravessada por dois tubos de náilon. Em um deles, procedia-se à redução da pressão interna do tubo e, no outro, ficava conectada uma seringa pela qual se coletava a solução.

Para extrair a solução do solo cerca de 12 horas após a fertirrigação, era reduzida a pressão interna do tubo (-80 kPa), com o auxílio de uma bomba de vácuo manual, e cerca de seis horas após este procedimento era efetuada a coleta da solução. Foram instalados 20 extratores de solução em toda a área do experimento, sendo um por parcela, na profundidade de $30 \mathrm{~cm}$, a $20 \mathrm{~cm}$ de distância 
horizontal de um emissor e sob a projeção da copa das árvores. A coleta da solução do solo foi realizada no final do mês de abril dos anos de 2008, 2009 e 2011.

A produção foi determinada através da massa e do número de frutos, sendo efetuada a multiplicação do número de frutos das 10 plantas úteis por parcela, pela massa média dos frutos, obtendo-se a produtividade em kg planta ${ }^{-1}$. Este valor foi multiplicado pelo número de plantas por hectare, obtendo-se assim a produtividade em $\mathrm{t} \mathrm{ha}^{-1}$. Para a obtenção da massa média de frutos, foram coletados 40 frutos das 10 plantas úteis de cada parcela, sendo quatro por planta e um por quadrante. A colheita da laranjeira Valência, que é de ciclo tardio, foi realizada aproximadamente no mês de outubro de cada safra.

Os dados foram submetidos à análise de variância e regressão, utilizando o programa estatístico Sistema para Análise de Variância - SISVAR (FERREIRA, 2011). Estudos de correlação foram feitos entre os resultados da análise de solo, solução do solo e a produção.

\section{RESULTADOS E DISCUSSÃO}

\section{Valores de pH e nutrientes no solo e na solução do solo}

Tanto o solo como a solução do solo apresentaram redução nos valores de $\mathrm{pH}$ em função das doses de $\mathrm{N}, \mathrm{P}_{2} \mathrm{O}_{5}$ e $\mathrm{K}_{2} \mathrm{O}$ aplicadas, para todos os anos avaliados (Tabelas $1 \mathrm{e} 2$ ). Isso ocorreu, pois, as fontes de $\mathrm{N}$ e $\mathrm{P}$ utilizadas, nitrato de amônio e ácido fosfórico, respectivamente, pos suem caráter ácido. Além disso, a fertirrigação efetuada via gotejo faz com que o fertilizante fique concentrado em um volume de solo restrito, potencializando o efeito acidificante das adubações. A redução do pH do solo e da solução do solo em sistema de fertirrigação por gotejamento também foi observada por ZANINI et al. (2007), SOUZA et al. (2012) e TEIXEIRA et al. (2007), que trabalharam com fertilizantes de reação ácida.

Considerando todos os tratamentos e anos de avaliação, o pH do solo variou de 5,7 a 4,1, enquanto o pH da solução do solo foi de 8,5 a 2,9 (Tabelas 1 e 2). Portanto, o pH do solo e da solução do solo variaram 1,6 e 5,6 unidades, respectivamente, refletindo a sensibilidade da análise da solução do solo. $\mathrm{O}$ pH da solução do solo, considerando apenas o tratamento que não recebeu fertilizante, aumentou de 5,1 para 7,7, entre os anos de 2008 e 2011 (Tabela 2), devido, provavelmente, às calagens realizadas em todas as safras, porém os valores de $\mathrm{pH}$ do solo, considerando o mesmo tratamento e período, mostraram menor variação $(5,1$ a 5,7).

No primeiro ano do experimento, os valores de $\mathrm{P}$ no solo e na solução do solo aumentaram de acordo com as doses de P aplicadas. Porém, no segundo ano, a solução do solo refletiu melhor os tratamentos (Tabelas 1 e 2). Valores de $\mathrm{P}$ no solo acima de $30 \mathrm{mg} \mathrm{dm}^{-3}$ são considerados altos para a cultura de citros, de acordo com QUAGGIO et al. (2005). No início do experimento, os valores de $\mathrm{P}$ no solo já estavam altos $\left(57 \mathrm{mg} \mathrm{dm}^{-3}\right)$, por isso que, na última safra, não foi aplicado $\mathrm{P}$.

Os valores de $\mathrm{P}$ no solo foram muito maiores que os observados na solução solo, fato já esperado devido ao comportamento do $\mathrm{P}$ nos solos tropicais. Considerando todo o período experimental e os tratamentos, os valores de $\mathrm{P}$ no solo variaram ao redor de três vezes (32 a $97 \mathrm{mg} \mathrm{dm}^{-3}$ ), enquanto na solução do solo essa variação foi de aproximadamente 200 vezes $\left(0,06\right.$ a 11,59 $\mathrm{mg} \mathrm{L}^{-1}$ ) (Tabelas 1 e 2). RAIJ (2011) relata que os valores de concentração de fósforo na solução do solo normalmente são baixos, em torno de $0,1 \mathrm{mg} \mathrm{L}^{-1}$, e que isso é decorrente da baixa solubilidade dos compostos de fósforo existentes no solo e da alta capacidade de adsorção do elemento pelas partículas do solo. SOUZA et al. (2012), em cultivo fertirrigado de laranjeira, observaram que os valores de $\mathrm{P}$ na solução do solo chegaram a $13,4 \mathrm{mg} \mathrm{L}^{-1}$. Como na fertirrigação por gotejamento os fertilizantes são aplicados em uma área restrita do solo, os sítios de fixação de $\mathrm{P}$ são preenchidos rapidamente, proporcionando aumento na disponibilidade deste nutriente. Esta situação também foi observada por ZANINI et al. (2007) e LAURINDO et al. (2010).

$\mathrm{Na}$ solução do solo, os valores de $\mathrm{K}$ aumentaram de acordo com as doses de fertilizantes aplicadas e variaram entre 0,01 e $2,86 \mathrm{mmol}_{\mathrm{c}} \mathrm{L}^{-1}$, considerando todos os tratamentos e anos de análise, o que representa uma variação maior que 250 vezes (Tabela 2). Nos anos de 2008 e 2009, o 
teor de K na solução do solo foi maior que no solo, nos tratamentos que receberam as maiores doses de nutrientes (Tabelas 1e 2), fato que chama bastante a atenção, pois de acordo com RAIJ (2011), o teor de $\mathrm{K}$ na solução do solo pouco representa em relação ao teor trocável do solo. Também é interessante observar que, nas maiores doses de fertilizantes, a concentração de $\mathrm{K}$ na solução foi maior que a soma dos valores de Ca e Mg na solução (Tabela 2). De acordo com MIRANDA et al. (2010), quando a quantidade de potássio no solo é relativamente pequena, em relação à capacidade de troca de cátions, a adsorção de potássio é controlada principalmente pela concentração de potássio na solução do solo; portanto, aumentando a concentração de potássio na solução, a capacidade tampão do potássio decresce.

Os valores de $\mathrm{K}$ no solo permaneceram baixos para a cultura de citros de acordo com QUAGGIO et al. (2005), menores que 1,5 $\mathrm{mmol}_{\mathrm{c}} \mathrm{dm}^{-3}$, com exceção do tratamento que recebeu a dose mais elevada de nutriente, nos anos de 2008 e 2011 (Tabela 1). O teor de K no solo aumentou de acordo com as doses de fertilizantes aplicadas e variou em torno de 2,5 vezes $\left(0,7\right.$ a $1,8 \mathrm{mmol}_{\mathrm{c}}$ $\mathrm{dm}^{-3}$ ), considerando todos os tratamentos e o período de análise. TEIXEIRA et al. (2007) trabalharam com a aplicação de K em bananeira, via adubação sólida e fertirrigação, e observaram baixa concentração de K no solo no sistema fertirrigado, que foi atribuída a perdas por lixiviação. Segundo os autores, em solos com significativa movimentação de água no perfil, a perda de água por percolação advinda da chuva durante ano é muito grande, principalmente em cultivos irrigados, em que há maior disponibilidade de água.

TABELA 1. Análise de solo, para os anos de 2008, 2009 e 2011, na camada de 0-40 cm, em função de doses de $\mathrm{N}, \mathrm{P}_{2} \mathrm{O}_{5}$ e $\mathrm{K}_{2} \mathrm{O}$. Soil analysis for the years of 2008, 2009 and 2011, at 0 $40 \mathrm{~cm}$ layer, according to the $\mathrm{N}, \mathrm{P}_{2} \mathrm{O}_{5}$ and $\mathrm{K}_{2} \mathrm{O}$ doses.

\begin{tabular}{|c|c|c|c|c|c|c|c|c|c|c|}
\hline \multirow{2}{*}{ Trat. } & $\mathrm{pH}$ & $\mathrm{K}$ & $\mathrm{Ca}$ & $\mathrm{Mg}$ & $\mathrm{P}$ & $\mathrm{B}$ & $\mathrm{Cu}$ & $\mathrm{Fe}$ & $\mathrm{Mn}$ & $\mathrm{Zn}$ \\
\hline & $\mathrm{CaCl}_{2}$ & \multicolumn{3}{|c|}{---- $\mathrm{mmol}_{\mathrm{c}} \mathrm{dm}^{-3}$---- } & \multicolumn{6}{|c|}{ 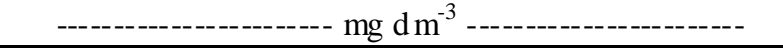 } \\
\hline \multicolumn{11}{|c|}{ ( } \\
\hline $0 \%$ & 5,2 & 1,1 & 18 & 6 & 47 & 0,5 & 1,4 & 28 & 6,1 & 3,7 \\
\hline $25 \%$ & 5,0 & 1,1 & 16 & 5 & 32 & 1,3 & 1,3 & 37 & 7,0 & 4,5 \\
\hline $50 \%$ & 4,4 & 1,3 & 13 & 4 & 45 & 1,1 & 1,4 & 41 & 7,6 & 4,8 \\
\hline $100 \%$ & 4,5 & 1,3 & 14 & 5 & 60 & 1,4 & 1,5 & 51 & 7,4 & 4,3 \\
\hline $200 \%$ & 4,1 & 1,8 & 11 & 3 & 67 & 1,7 & 1,3 & 63 & 10,9 & 7,3 \\
\hline $\begin{array}{c}\text { Regressão } \\
\mathbf{R}^{2}\end{array}$ & $\begin{array}{l}\mathrm{L}^{* *} \\
\mathbf{0 , 8 2}\end{array}$ & $\begin{array}{l}\mathrm{L}^{* *} \\
\mathbf{0 , 8 8}\end{array}$ & ns & ns & $\begin{array}{c}\mathrm{L}^{*} \\
0,72\end{array}$ & ns & ns & $\begin{array}{l}\mathrm{L}^{* * *} \\
\mathbf{0 , 9 7}\end{array}$ & ns & ns \\
\hline \multicolumn{11}{|l|}{2009} \\
\hline $0 \%$ & 5,7 & 0,7 & 24 & 10 & 67 & 0,5 & 1,5 & 40 & 4,5 & 4,0 \\
\hline $25 \%$ & 5,1 & 1,0 & 23 & 9 & 83 & 0,7 & 1,9 & 76 & 6,2 & 9,3 \\
\hline $50 \%$ & 4,7 & 1,2 & 13 & 6 & 65 & 0,8 & 1,7 & 82 & 15,3 & 9,0 \\
\hline $100 \%$ & 4,4 & 1,3 & 16 & 5 & 86 & 0,8 & 1,8 & 78 & 10,8 & 9,3 \\
\hline $200 \%$ & 4,2 & 1,3 & 15 & 6 & 97 & 0,9 & 1,7 & 89 & 9,2 & 8,9 \\
\hline Regressão & $\mathrm{L}^{* *}$ & $\mathrm{Q}^{* *}$ & $\mathrm{~L}^{* *}$ & $\mathrm{Q}^{*}$ & & $\mathrm{~L}^{*}$ & & $\mathrm{~L}^{* *}$ & $\mathrm{Q}^{* *}$ & \\
\hline $\mathrm{R}^{2}$ & 0,76 & 0,96 & 0,43 & 0,93 & ns & 0,62 & ns & 0,49 & 0,55 & ns \\
\hline \multicolumn{11}{|l|}{2011} \\
\hline $0 \%$ & 5,1 & 0,7 & 18 & 7 & 62 & 0,4 & 3,3 & 47 & 6,6 & 5,0 \\
\hline $25 \%$ & 5,1 & 0,8 & 19 & 9 & 64 & 0,5 & 3,6 & 59 & 6,8 & 7,4 \\
\hline $50 \%$ & 5,1 & 0,9 & 21 & 11 & 48 & 0,5 & 3,5 & 50 & 7,5 & 6,2 \\
\hline $100 \%$ & 4,5 & 1,1 & 14 & 7 & 91 & 0,6 & 3,9 & 91 & 9,7 & 8,7 \\
\hline $200 \%$ & 4,3 & 1,7 & 17 & 10 & 76 & 0,6 & 3,5 & 91 & 8,7 & 10,1 \\
\hline Regressão & $\mathrm{L}^{* *}$ & $\mathrm{~L}^{* *}$ & & & & & & $\mathrm{~L}^{* *}$ & & $\mathrm{~L}^{*}$ \\
\hline $\mathrm{R}^{2}$ & 0,89 & 0,99 & ns & ns & ns & ns & ns & 0,72 & $\mathrm{~ns}$ & 0,83 \\
\hline \multicolumn{11}{|l|}{ Teste F } \\
\hline Trat. (T) & $* *$ & $* *$ & $*$ & $\mathrm{~ns}$ & $* *$ & $* *$ & $\mathrm{~ns}$ & $* *$ & $*$ & $*$ \\
\hline Ano (A) & ns & $* *$ & $*$ & $* *$ & $* *$ & $* *$ & $* *$ & $* *$ & $\mathrm{~ns}$ & $*$ \\
\hline $\mathrm{T} \times \mathrm{A}$ & $\mathrm{ns}$ & $*$ & $\mathrm{~ns}$ & $\mathrm{~ns}$ & $\mathrm{~ns}$ & $*$ & ns & $\mathrm{ns}$ & $\mathrm{ns}$ & ns \\
\hline
\end{tabular}


Para o ano de 2011, muitos dos nutrientes avaliados não apresentaram variação significativa em função dos tratamentos, tanto no solo como na solução do solo (Tabelas 1 e 2). Isso pode ter ocorrido, pois foi um ano muito chuvoso, apenas no período entre janeiro e abril de 2011 a precipitação foi de $1020 \mathrm{~mm}$. Porém, os nutrientes que foram aplicados via fertirrigação ( $\mathrm{N}$ e $\mathrm{K}$ ) e os valores de $\mathrm{pH}$ e condutividade elétrica $(\mathrm{CE})$, variaram de forma significativa $(\mathrm{p}<0,01)$ em relação aos tratamentos, neste ano (P não foi aplicado em 2011).

A redução dos valores de $\mathrm{pH}$ observada no solo e na solução do solo, de acordo com as doses de $\mathrm{N}, \mathrm{P}_{2} \mathrm{O}_{5}$ e $\mathrm{K}_{2} \mathrm{O}$ aplicadas, veio acompanhada do aumento dos teores de alguns micronutrientes (Tabelas 1 e 2), e isso ocorreu devido ao aumento da disponibilidade destes elementos em ambientes com valores de $\mathrm{pH}$ baixo (RAIJ, 2011). Tal resultado também foi observado por SOUZA et al. (2012), que trabalharam com aplicação de fertilizantes ácidos (nitrato de amônio e ácido fosfórico) via fertirrigação na citricultura.

TABELA 2. Análise da solução do solo, para os anos de 2008, 2009 e 2011, na profundidade de $30 \mathrm{~cm}$, em função das doses de $\mathrm{N}, \mathrm{P}_{2} \mathrm{O}_{5}$ e $\mathrm{K}_{2} \mathrm{O}$. Soil solution analysis for the years of 2008, 2009 and 2011, at $30 \mathrm{~cm}$ depth, according to the $\mathrm{N}, \mathrm{P}_{2} \mathrm{O}_{5}$ and $\mathrm{K}_{2} \mathrm{O}$ doses.

\begin{tabular}{|c|c|c|c|c|c|c|c|c|c|c|}
\hline \multirow{2}{*}{ Trat. } & $\mathrm{pH}$ & K & $\mathrm{Ca}$ & $\mathrm{Mg}$ & $\mathrm{P}$ & $\mathrm{B}$ & $\mathrm{Cu}$ & $\mathrm{Fe}$ & $\mathrm{Mn}$ & $\mathrm{Zn}$ \\
\hline & $\mathrm{CaCl}_{2}$ & \multicolumn{3}{|c|}{$-\cdots \mathrm{mmol}_{\mathrm{c}} \mathrm{L}^{-1} \ldots$} & \multicolumn{6}{|c|}{ - } \\
\hline \multicolumn{11}{|c|}{ 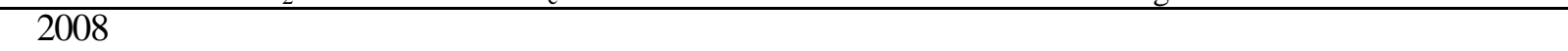 } \\
\hline $0 \%$ & 5,1 & 0,11 & 0,38 & 0,25 & 1,49 & 0,45 & 0,17 & 0,01 & 0,48 & 1,16 \\
\hline $25 \%$ & 5,3 & 0,31 & 1,02 & 0,50 & 1,46 & 2,33 & 0,10 & 0,01 & 1,64 & 1,97 \\
\hline $50 \%$ & 3,6 & 1,21 & 1,93 & 0,49 & 1,50 & 4,90 & 0,33 & 0,01 & 7,94 & 7,90 \\
\hline $100 \%$ & 3,1 & 2,86 & 1,35 & 0,44 & 5,57 & 8,65 & 0,48 & 0,01 & 8,94 & 10,39 \\
\hline $200 \%$ & 3,0 & 2,39 & 0,88 & 0,40 & 11,59 & 5,79 & 0,45 & 0,01 & 7,73 & 8,18 \\
\hline Regressão & $\mathrm{L}^{* *}$ & $\mathrm{Q}^{* *}$ & $\mathrm{Q}^{* *}$ & $\mathrm{Q}^{*}$ & $\mathrm{~L}^{* *}$ & $\mathrm{Q}^{* *}$ & $\mathrm{~L}^{*}$ & \multirow{2}{*}{ ns } & $\mathrm{Q} * *$ & $\mathrm{Q} * *$ \\
\hline $\mathrm{R}^{2}$ & 0,66 & 0,92 & 0,66 & 0,50 & 0,90 & 0,97 & 0,64 & & 0,89 & 0,91 \\
\hline \multicolumn{11}{|l|}{2009} \\
\hline $0 \%$ & 7,1 & 0,04 & 0,02 & 0,03 & 0,06 & 0,03 & 0,01 & 0,01 & 0,09 & 0,07 \\
\hline $25 \%$ & 6,4 & 0,12 & 0,09 & 0,07 & 0,96 & 0,12 & 0,02 & 0,01 & 0,23 & 0,26 \\
\hline $50 \%$ & 3,5 & 0,54 & 0,33 & 0,38 & 2,17 & 0,50 & 0,08 & 0,02 & 1,59 & 1,26 \\
\hline $100 \%$ & 3,0 & 0,65 & 0,24 & 0,27 & 5,30 & 0,58 & 0,05 & 0,03 & 1,54 & 1,89 \\
\hline $200 \%$ & 2,9 & 2,83 & 0,23 & 0,28 & 8,43 & 0,39 & 0,04 & 0,06 & 5,51 & 7,33 \\
\hline Regressão & $\mathrm{L}^{* *}$ & $\mathrm{~L}^{* *}$ & & $\mathrm{Q}^{*}$ & $\mathrm{~L}^{* *}$ & & & $\mathrm{~L}^{* * *}$ & $\mathrm{~L}^{* *}$ & $\mathrm{~L}^{* *}$ \\
\hline $\mathrm{R}^{2}$ & 0,63 & 0,92 & ns & 0,65 & 0,98 & ns & ns & 0,99 & 0,93 & 0,94 \\
\hline \multicolumn{11}{|l|}{2011} \\
\hline $0 \%$ & 7,7 & 0,01 & 0,23 & 0,05 & 0,68 & 1,30 & 3,52 & 0,01 & 1,53 & 3,10 \\
\hline $25 \%$ & 8,5 & 0,01 & 0,09 & 0,12 & 0,69 & 1,17 & 3,44 & 0,01 & 1,00 & 3,25 \\
\hline $50 \%$ & 6,3 & 0,03 & 0,06 & 0,07 & 0,62 & 1,22 & 3,84 & 0,01 & 1,38 & 3,18 \\
\hline $100 \%$ & 3,8 & 0,22 & 0,23 & 0,22 & 0,36 & 1,29 & 3,80 & 0,01 & 3,03 & 3,53 \\
\hline $200 \%$ & 3,3 & 0,25 & 0,07 & 0,08 & 1,25 & 1,52 & 3,94 & 0,01 & 1,35 & 3,61 \\
\hline $\begin{array}{c}\text { Regressão } \\
\mathrm{R}^{2}\end{array}$ & $\begin{array}{l}\mathrm{L} * * \\
0,79\end{array}$ & $\begin{array}{l}\mathrm{L}^{* *} \\
0,83\end{array}$ & ns & ns & ns & ns & $\begin{array}{l}\mathrm{L}^{* *} \\
0,67\end{array}$ & $\mathrm{~ns}$ & ns & $\mathrm{ns}$ \\
\hline \multicolumn{11}{|l|}{ Teste $\mathrm{F}$} \\
\hline Trat. (T) & $* *$ & $* *$ & $* *$ & $* *$ & $* *$ & $* *$ & $* *$ & $\mathrm{~ns}$ & $* *$ & $* *$ \\
\hline Ano (A) & $* *$ & $* *$ & $* *$ & $* *$ & $* *$ & $* *$ & $* *$ & $* *$ & $* *$ & $* *$ \\
\hline $\mathrm{T} \times \mathrm{A}$ & $* *$ & $* *$ & ** & ns & $* *$ & $* *$ & ns & * & $* *$ & $* *$ \\
\hline
\end{tabular}

Trat. - tratamento; L - ajuste linear; Q - ajuste quadrático; $* * \mathrm{p}<0,01 ; * \mathrm{p}<0,05 ;$ ns - não significativo.

Os valores de B na solução do solo quase sempre foram maiores que os observados no solo, principalmente nos anos de 2008 e 2011 (Tabelas 1 e 2). Como a forma dominante deste elemento na solução do solo é o ácido bórico, que não possui carga, este nutriente não faz parte do complexo de troca. Por outro lado, os micronutrientes $\mathrm{Cu}, \mathrm{Mn}$ e $\mathrm{Zn}$ foram observados em maiores concentrações no solo, com algumas exceções nas maiores doses de fertilizantes (Tabelas 1 e 2). Como estes micronutrientes são cátions, ficam adsorvidos ao solo. Para o $\mathrm{Fe}$, foram observadas baixas concentrações na solução do solo e altas no solo; portanto, a técnica de análise da solução do solo pode não ser adequada para avaliar a disponibilidade deste nutriente, conforme já observado 
por SOUZA et al. (2012). Outra hipótese seria a de que o extrator DTPA estaria superestimando o teor de Fe no solo; porém em todos os anos avaliados, o teor de Fe nas folhas da laranjeira foi considerado adequado ou ficou um pouco acima do adequado de acordo com QUAGGIO et al. (2005) (dados não apresentados).

\section{Nitrogênio na solução do solo}

Devido à dinâmica do nitrogênio no solo, a análise do solo para este nutriente demanda cuidados, como a secagem ou o congelamento imediato das amostras logo após sua coleta. Diferentemente da solução do solo, o nitrogênio pode ser determinado diretamente no campo sem a necessidade de cuidados prévios (COELHO et al, 2014). A concentração de amônio $\left(\mathrm{N}-\mathrm{NH}_{4}\right)$ na solução do solo aumentou de acordo com as doses de $\mathrm{N}, \mathrm{P}_{2} \mathrm{O}_{5}$ e $\mathrm{K}_{2} \mathrm{O}$ aplicadas; no entanto, o nitrato $\left(\mathrm{N}-\mathrm{NO}_{3}\right)$ apresentou ajuste quadrático em função dos tratamentos. Independente mente da forma do $\mathrm{N}$, este elemento correlacionou-se de forma positiva com os tratamentos $(\mathrm{p}<0,01)$ em todos os anos de análise (Tabela 3). Isso mostra que a análise da solução do solo pode ser um bom indicativo da disponibilidade de $\mathrm{N}$ no solo para as plantas. Os valores de condutividade elétrica (CE) também se correlacionaram de forma positiva com a dose de fertilizante aplicada (Tabela 3).

Altos valores de $\mathrm{N}_{-} \mathrm{NH}_{4}$ foram observados na solução do solo nos tratamentos que receberam as maiores doses de fertilizantes (Tabela 3). Segundo SOUZA et al. (2012), valores de $\mathrm{N}-\mathrm{NH}_{4}$ na solução do solo acima de $2,9 \mathrm{mmol}_{\mathrm{c}} \mathrm{L}^{-1}$ podem ser tóxicos para a cultura de citros. Os autores comentam que fontes amoniacais, quando aplicadas via fertirrigação por gotejo, acidificam o solo, devido à aplicação localizada e ao processo de nitrificação. Após algum tempo, a acidez gerada interfere na atividade das bactérias repensáveis pela nitrificação, diminuindo a conversão do amônio a nitrato. Consequentemente, a concentração de amônio aumenta no solo, as plantas passam a absorver mais amônio e eliminam prótons $\left(\mathrm{H}^{+}\right)$, para manter o equilíbrio celular, acidificando ainda mais o ambiente. $\mathrm{O}$ excesso de amônio absorvido pela planta pode chegar a níveis tóxicos e causar danos ao desenvolvimento e à produção, além de diminuir a absorção de outros cátions, principalmente $\mathrm{Ca}$ (QUAGGIO et al., 2014).

TABELA 3. Valores de condutividade elétrica (CE), amônio e nitrato na solução do solo, para os anos de 2008, 2009 e 2011, na profundidade de $30 \mathrm{~cm}$, em função das doses de N, $\mathrm{P}_{2} \mathrm{O}_{5}$ e $\mathrm{K}_{2} \mathrm{O}$. Electrical conductivity (EC), ammonium and nitrate values in soil solution for the years of 2008,2009 and 2011 , at $30-\mathrm{cm}$ depth, according to the $\mathrm{N}, \mathrm{P}_{2} \mathrm{O}_{5}$ and $\mathrm{K}_{2} \mathrm{O}$ doses.

\begin{tabular}{|c|c|c|c|c|c|c|c|c|c|}
\hline \multirow{3}{*}{ Trat. } & \multicolumn{3}{|c|}{$\mathrm{CE}$} & \multicolumn{3}{|c|}{$\mathrm{N}-\mathrm{NH}_{4}$} & \multicolumn{3}{|c|}{$\mathrm{N}-\mathrm{NO}_{3}$} \\
\hline & 2008 & 2009 & 2011 & 2008 & 2009 & 2011 & 2008 & 2009 & 2011 \\
\hline & \multicolumn{3}{|c|}{--o- dS m ${ }^{-1}$------ } & \multicolumn{3}{|c|}{----- mmol $_{\mathrm{c}} \mathrm{L}^{-1}$} & \multicolumn{3}{|c|}{----- mmol $_{\mathrm{c}} \mathrm{L}^{-1}$------ } \\
\hline $0 \%$ & 0,1 & 0,1 & 0,3 & 1,3 & 0,1 & 0,2 & 0,5 & 0,2 & 0,3 \\
\hline $25 \%$ & 0,3 & 0,2 & 0,4 & 0,4 & 0,2 & 0,2 & 0,9 & 0,8 & 0,4 \\
\hline $50 \%$ & 0,7 & 0,3 & 0,5 & 0,6 & 0,7 & 0,2 & 3,0 & 3,5 & 0,6 \\
\hline $100 \%$ & 1,5 & 0,4 & 1,2 & 4,1 & 1,5 & 1,2 & 8,2 & 9,3 & 7,4 \\
\hline $200 \%$ & 1,5 & 1,2 & 1,0 & 4,5 & 2,4 & 4,8 & 6,4 & 5,1 & 6,8 \\
\hline Regressão & $\mathrm{Q}^{* *}$ & $\mathrm{~L}^{* *}$ & $\mathrm{Q}^{* *}$ & $\mathrm{~L}^{* *}$ & $\mathrm{~L}^{* *}$ & $\mathrm{~L}^{* *}$ & $\mathrm{Q}^{* *}$ & $\mathrm{Q}^{* *}$ & $\mathrm{Q}^{* *}$ \\
\hline $\mathrm{R}^{2}$ & 0,94 & 0,94 & 0,86 & 0,72 & 0,98 & 0,91 & 0,88 & 0,86 & 0,80 \\
\hline \multicolumn{10}{|l|}{ Teste F } \\
\hline Trat. (T) & & $* *$ & & & $* *$ & & & $* *$ & \\
\hline Ano (A) & & $* *$ & & & * & & & $* *$ & \\
\hline $\mathrm{T} \times \mathrm{A}$ & & $* *$ & & & $\mathrm{~ns}$ & & & $* *$ & \\
\hline
\end{tabular}

\section{Produtividade e correlações}

A produtividade de frutos apresentou ajuste quadrático em relação às doses de nutrientes aplicadas, sendo o valor máximo encontrado de $45 \mathrm{t} \mathrm{ha}^{-1}$, com a aplicação de $125 \%$ da dose de $\mathrm{N}$, $\mathrm{P}_{2} \mathrm{O}_{5}$ e $\mathrm{K}_{2} \mathrm{O}$, para a média dos anos de 2008, 2009 e 2011 (Figura 1). Os valores de $\mathrm{pH}, \mathrm{K}, \mathrm{P}, \mathrm{B}, \mathrm{Cu}$, $\mathrm{Mn}$ e $\mathrm{Zn}$ no solo e na solução do solo apresentaram comportamento semelhante, quando 
correlacionados com a produção (Tabela 4). Porém, para os valores de Ca e $\mathrm{Mg}$, o comportamento foi oposto, estes nutrientes no solo mostraram correlação ne gativa com a produção, e na solução do solo a correlação foi positiva. Para o Fe, o coeficiente de correlação entre os valores presentes no solo e a produção foi de $0,84(\mathrm{p}=0,073)$, enquanto na solução do solo o coeficiente foi baixo $\left(R^{2}=0,51\right.$ e $\left.p=0,376\right)$.

Como os elementos no solo e na solução do solo estão em equilíbrio, quando correlacionadas as variáveis avaliadas no solo com as mesmas avaliadas na solução do solo, o ajuste foi alto para os valores de $\mathrm{pH}, \mathrm{K}, \mathrm{P}, \mathrm{Fe}, \mathrm{Mn}$ e Zn. Porém, para os teores de B e, principalmente, de $\mathrm{Cu}$, a correlação foi baixa (Tabela 4). No caso do Fe, é importante destacar que, para a média dos anos avaliados, a correlação foi alta e positiva; porém, quando feita anualmente, apenas no ano de 2009 os valores correlacionaram-se. Valores de correlação negativos e baixos foram observados para os teores de $\mathrm{Ca}$ e $\mathrm{Mg}$ (Tabela 4). Na solução do solo, a quantidade de $\mathrm{Ca}$ e $\mathrm{Mg}$ apresentou tendência de aumento e no solo de redução, em função das doses de $\mathrm{N}, \mathrm{P}_{2} \mathrm{O}_{5}$ e $\mathrm{K}_{2} \mathrm{O}$, principalmente nos anos de 2008 e 2009 (Tabelas 1 e 2). Ca e Mg foram fornecidos apenas via calagem e em quantidades iguais para todos os tratamentos. Devido à acidificação e atraídos pelo gradiente de concentração, $\mathrm{Ca}$ e $\mathrm{Mg}$ podem ter-se deslocado do solo para a solução. ERNANI et al. (2007) observaram que a adição de cloreto de potás sio aumentou a percolação de $\mathrm{Ca}$ e $\mathrm{Mg}$ no solo. Os autores também comentaram que reações secundárias decorrentes da adição de adubos ao solo são pouco estudadas. DELBEM et al. (2011) observaram diminuição dos teores de $\mathrm{Ca}$ e $\mathrm{Mg}$ no so lo, resultante da lixiviação, devido aos baixos valores de $\mathrm{pH}$ do solo, provocado pelo uso de adubos nitrogenados, como ureia e sulfato de amônio.

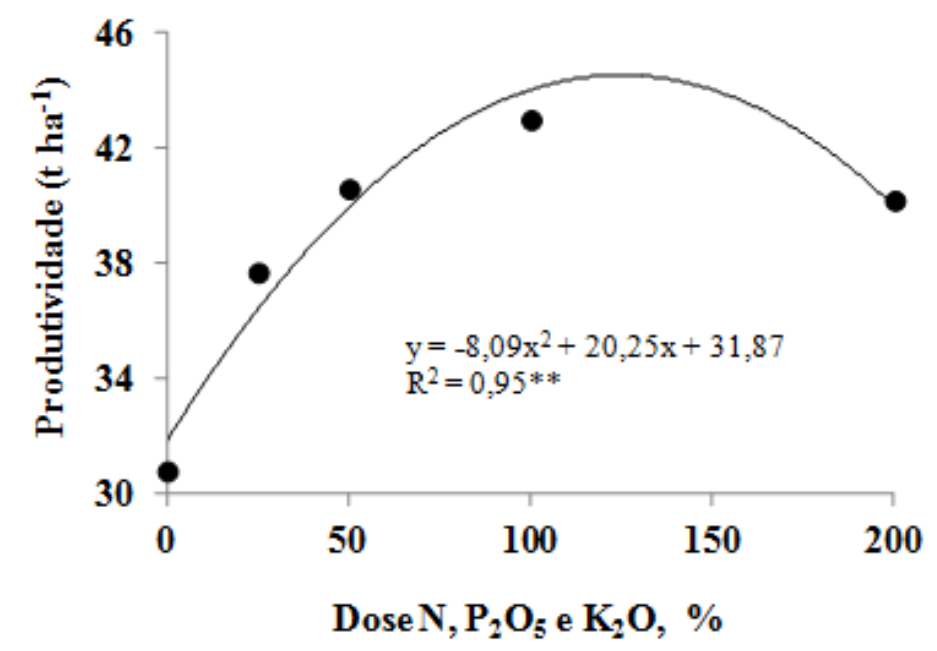

FIGURA 1. Produtividade de frutos em função das doses de $\mathrm{N}, \mathrm{P}_{2} \mathrm{O}_{5}$ e $\mathrm{K}_{2} \mathrm{O}$, para média dos anos de 2008, 2009 e 2011 (** $\mathrm{p}<0,01)$. Fruit yield as a function of $\mathbf{N}, \mathbf{P}_{2} \mathbf{O}_{5}$ and $\mathrm{K}_{2} \mathrm{O}$ doses for means of the years of 2008, 2009 and 2011 (** $p<0.01$ ). 
TABELA 4. Valores de coeficiente de determinação, entre os resultados de pH e os teores de nutrientes no solo (S), na solução do solo (SS) e a produtividade (Prod.), para média dos anos de 2008, 2009 e 2011. Coefficient of determination values between pH and nutrient contents in both soil and soil solution, and yield for means of the years of 2008, 2009 and 2011.

\begin{tabular}{|c|c|c|c|c|c|c|c|}
\hline Variáveis & $\mathrm{S} v s . \mathrm{SS}$ & $\begin{array}{c}\text { Equação } \\
y=S \quad x=S S\end{array}$ & $\mathrm{p}$ & Prod.vs. S & $\mathrm{p}$ & Prod. vs. SS & $\mathrm{p}$ \\
\hline $\mathrm{pH}$ & 0.96 & $y=0,25 x+3,60$ & 0,011 & -0.82 & 0,087 & -0.79 & 0,112 \\
\hline $\mathrm{K}$ & 0.97 & $y=0,38 x+0,86$ & 0,006 & 0.67 & 0,213 & 0.68 & 0,207 \\
\hline $\mathrm{Ca}$ & -0.63 & $y=-7,71 x+20,47$ & 0,258 & -0.86 & 0,064 & 0.78 & 0,118 \\
\hline $\mathrm{Mg}$ & -0.67 & $y=-7,06 x+8,58$ & 0,214 & -0.79 & 0,113 & 0.97 & 0,006 \\
\hline $\mathrm{P}$ & 0.87 & $y=4,10 x+54,49$ & 0,057 & 0.49 & 0,401 & 0.52 & 0,370 \\
\hline B & 0.78 & $y=0,15 x+0,52$ & 0,123 & 0.86 & 0,060 & 0.92 & 0,028 \\
\hline $\mathrm{Cu}$ & 0.29 & $y=0,27 x+1,85$ & 0,635 & 0.79 & 0,115 & 0.73 & 0,160 \\
\hline $\mathrm{Fe}$ & 0.86 & $y=2041,43 x+30,23$ & 0,065 & 0.84 & 0,073 & 0.51 & 0,376 \\
\hline $\mathrm{Mn}$ & 0.93 & $y=0,92 x+5,60$ & 0,023 & 0.86 & 0,059 & 0.83 & 0,085 \\
\hline $\mathrm{Zn}$ & 0.81 & $y=0,63 x+4,42$ & 0,095 & 0.82 & 0,087 & 0.79 & 0,116 \\
\hline
\end{tabular}

$\mathrm{p}-$ valor de $\mathrm{p}$ ( $\mathrm{p}$ value).

\section{CONCLUSÕES}

Em sistemas de fertirrigação por gotejo, a quantidade de nutrientes presentes no bulbo molhado normalmente supera os valores de nutrientes na solução do solo tradicionalmente conhecidos na literatura.

As fontes e as doses de $\mathrm{N}, \mathrm{P}_{2} \mathrm{O}_{5}$ e $\mathrm{K}_{2} \mathrm{O}$ aplicadas proporcionaram redução nos valores de $\mathrm{pH}$ e aumento dos teores de $\mathrm{P}$ e $\mathrm{K}$ no solo e na solução do solo. Porém, a amplitude de variação dos valores de $\mathrm{pH}$ e dos teores de nutrientes foi maior na solução.

Os resultados sugerem que a análise da solução do solo pode ser utilizada como ferramenta para estimar os valores de $\mathrm{pH}, \mathrm{P}, \mathrm{K}, \mathrm{Mn}$ e $\mathrm{Zn}$ no solo, e também para determinar a disponibilidade de nitrogênio em sistemas de fertirrigação na citricultura.

\section{AGRADECIMENTOS}

À Fundação de Amparo à Pesquisa do Estado de São Paulo pela concessão de bolsa e auxílio pesquisa, e à empresa Citrovita (Grupo Votorantim), pela contribuição no desenvolvimento deste ensaio.

\section{REFERÊNCIAS}

ALLEN, R.G.; PEREIRA, L.S.; RAES, D.; SMITH, M. Crop evapotranspiration - guidelines for computing crop water require ments. Roma: FAO, 1998. 300p. (Irrigation and Drainage, Paper 56).

COELHO, E.F.; COSTA, F.C.; SILVA, A.C.A.; CARVALHO, G.C. Concentração de nitrato no perfil do solo fertigado com diferentes concentrações e fontes de nitrogênio. Revista Brasileira de Engenharia Agrícola e Ambiental, Campina Grande, v.18, n.3, p.263-269, mar. 2014.

COELHO, E.F.; COELHO FILHO, M.A. MAGALHÃES, A.F.J.; OLIVEIRA, A.S. Irrigação e fertirrigação na cultura do citros. In: SOUSA, V.F.; MAROUELLI, W.A.; COELHO, E.F.; PINTO, J.M.; COELHO FILHO, M.A. Irrigação e fertirrigação em frutíferas e hortaliças. Brasília: Embrapa Informação Tecnológica, 2011. p.415-439.

DELBEM, F.C.; SCABORA, M.H.; SOARES FILHO, C.V.; HEINRICHS, R.; CROCIOLLI, C.A.; CASSIOLATO, A.M.R. Fontes e doses de adubação nitrogenada na atividade microbiana e fertilidade do solo cultivado com Brachiaria brizantha. Acta Scientiarum. Agronomy, Maringá, v.33, n.2, p.361-367, abr./jun. 2011. 
ERNANI, P.R.; BAYER, C.; ALMEIDA, J.A.; CASSOL, P.C. Mobilidade vertical de cátions influenciada pelo método de aplicação de cloreto de potássio em solos com carga variável. Revista Brasileira de Ciência do Solo, Viçosa, MG, v.31, n.2, p.393-402, mar./abr. 2007.

FERREIRA, D. F. SISVAR: A computer statistical analysis system. Ciência e Agrotecnologia, Lavas, v.35, n.6, p.1039-1042, nov./dec. 2011.

LAURINDO, V.T.; SILVA, G.O.; PAVANI, L.C.; QUAGGIO, J.A. Padrão de distribuição de K, Ca, Mg e P no solo de um pomar de citros em função da fertirrigação. Engenharia Agrícola, Jaboticabal, v.30, n.5, p.909-921, set./out. 2010.

MEDEIROS, P.R.F.; DUARTE, S.N.; UYEDA, A.; SILVA, E.S.S.; MEDEIROS, J.F. Tolerância da cultura do tomate à salinidade do solo em ambiente protegido. Revista Brasileira de Engenharia Agrícola e Ambiental, Campina Grande, v.16, n.1, p.51-55, jan. 2012.

MIRANDA, J.H.; BÉRGAMO, L.R.; REIS, J.B.R.S.; CRUCIANI, D.E.; DUARTE, S.N. Distribuiçõa da concentração de potássio no solo em lisímetros cultivados com amendoim. Engenharia Agrícola, Jaboticabal, v.30, n.2, p.253-263, mar./abr. 2010.

OLIVEIRA, F.A.; MEDEIROS J.F.; DUARTE, S.N.; SILVA JR., N.J.; CAMPELO, C.M. Calibração de extratores providos de cápsula porosa para monitoramento da salinidade e da concentração de íons. Enge nharia Agrícola, Jaboticabal, v.31, n.3, p.520-528, maio/jun. 2011.

QUAGGIO, J.A.; SOUZA, T.R.; ZAMBROSI, F.C.B.; BOARETTO, R.M.; MATTOS JR., D. Nitrogenfertilizer forms affect nitrogen-use efficiency in fertigated citrus groves. Journal of Plant Nutrition and Soil Science, Weinhe im, v. 117, n.3, p.401 - 411, jun. 2014.

QUAGGIO, J.A.; MATTOS JR., D.; CANTARELLA, H. Manejo da fertilidade do solo na citricultura. In: MATTOS JR., D.; DE NEGRI., J.D.; PIO, R.M.; POMPEU JR., J., (Ed.) Citros. Campinas: Instituto Agronômico e Fundag, 2005. p.483-507.

RAIJ, B. Van. Fertilidade do solo e manejo de nutrientes. Piracicaba: International Plant Nutrition Institute, 2011. 420p.

RAIJ, B. Van; ANDRADE, J.C.; CANTARELLA, H.; QUAGGIO, J.A. Análise química para avaliação da fertilidade de solos tropicais. Campinas: Instituto Agronômico, 2001. 285p.

SANTOS, H.G.; JACOMINE, P.K.T.; ANJOS, L.H.C.; OLIVEIRA, V.A.; OLIVEIRA, J.B.; COELHO, M.R.; LUMBRERAS, J.F.; CUNHA, T.J.F. Sistema brasileiro de classificação de solos. 2.ed. Rio de Janeiro: Embrapa Solos, 2006. 306p.

SILVA JR. M.J.; DUARTE, S.N.; OLIVEIRA, F.A.; MEDEIROS, J.F.; DUTRA, I. Resposta do me loe iro à fertirrigação controlada através de íons na solução do solo: parâmetros produtivos. Revista Brasileira de Enge nharia Agrícola e Ambiental, Campina Grande, v.14, n.7, p.723-729, jul. 2010.

SOUZA, E.R.; MELO, H.F.; ALMEIDA, B.G.; MELO, D.V.M. Comparação de métodos de extração da solução do solo. Revista Brasileira de Engenharia Agrícola e Ambiental, Campina Grande, v.17, n.14, p.510-517, maio 2013.

SOUZA, T.R.; VILLAS BÔAS, R.L.; QUAGGIO, J.A.; SALOMÃO, L.C.; FORATTO, L.C. Dinâmica de nutrientes na solução do solo em pomar fertirrigado de citros. Pesquisa Agropecuária B rasileira, Brasîlia, v.47, n.6, p.846-854, jun. 2012.

TEIXEIRA, L.A.J.; NATALE, W.; BETTIOL NETO, J.M.; MARTINS, A.L.M. Nitrogênio e potássio em bananeira via fertirrigação e adubação convencional - atributos químicos do solo. Revista B rasileira de Fruticultura, Jaboticabal, v.29, n.1, p.143-152, abr. 2007.

ZANINI, J.R.; BARRETO, A.K.G.; FORATTO, L.C.; NATALE, W. Distribuição de fósforo no bulbo molhado, aplicado via fertirrigação por gotejamento com ácido fosfórico. Enge nharia Agrícola, Jaboticabal, v.27, n.1, p.180-193, jan./abr. 2007. 